\title{
When parameters collide: A warning about categorization models
}

\author{
J. DAVID SMITH \\ State University of New York, Buffalo, New York
}

\begin{abstract}
Similarity-choice (S-C) models of categorization contain two principal mathematical transformations: an exponential-decay similarity function and a choice rule. However, there is a tension between the psychological processes that models emulate and the mathematics they use to do so. To illustrate this, I show that in these models an unappreciated interaction occurs between the mathematical transformations so that the stages of the model essentially cancel each other out. The result is that the model's output reflects its input linearly. This cancellation phenomenon has potentially serious implications regarding the interpretation and use of S-C models. The phenomenon also raises questions about the simplification and psychological grounding of categorization models. Modelers broadly might benefit from an internal analysis of their models, such as that described here.
\end{abstract}

Mathematical models are part of theory building in cognitive science. At their best, models help us to instantiate, in parameter values, states and processes of mind and to evaluate psychological assumptions by testing their fit to data. However, there is a tension between the psychological processes that models emulate and the mathematics that they use to do so. Data transformations are crucial to modeling. We transform physical measures of stimulus relations into psychical measures, such as similarity. We transform psychical measures into predicted-performance measures. There is always the question of how faithful these transformations are to psychological representation and process. This question is sharpened because we ignore the psychological aspect at the critical time of evaluating a model's fit. This evaluation is purely mathematical, with fit measures comparing predicted performance and observed performance.

One goal of the present article is to illustrate this tension through the use of the influential class of similarity-choice (S-C) categorization models. I will show that an unappreciated interaction often occurs between the mathematical transformations in such a model so that the model's stages essentially cancel each other out. The result is that the model's output reflects its input linearly, despite the presumption of nonlinear psychological transformations. This has potentially serious implications regarding the interpretation and use of these models. This goal resonates with the concern expressed by others (e.g., Maddox \& Ashby, 1998) that the psychological grounding of $\mathrm{S}-\mathrm{C}$ models is tenuous.

The preparation of this article was supported by National Institutes of Health Grant HD-38051. Correspondence concerning this article should be addressed to J. D. Smith, Department of Psychology, 346 Park Hall, SUNY Buffalo, Buffalo, NY 14260 (e-mail: psysmith@buffalo.edu).
However, the present article also gives reason for optimism that we might simplify these models and make them more expressive of psychological process and representation.

The second goal of this article is to raise a more general issue about evaluating cognitive models. There is a tendency to evaluate models using an external criterion - that is, their fit to data - to index their success and strengthen their attendant theory (also see Roberts \& Pashler, 2000). This tendency has become stronger as models have become more complex, with internal structures that are difficult to query and understand. In contrast, in this article I emphasize that evaluating a model's internal structure and behavior is as important as evaluating its external fit, because a model's internal misbehavior can undermine its interpretative value as surely as poor fit can. Therefore, beyond fit, it is also important to ask whether or not (1) a model's components preserve their psychological meaning, (2) the processes presumably instantiated in the model are expressed by the model, and (3) the components of a model interact in a way that changes the model's character.

The ideas presented in this article apply equally to prototype and exemplar $\mathrm{S}-\mathrm{C}$ models. They neither promote nor are selectively critical of any representational theory of categorization (e.g., exemplar theory, prototype theory) or any body of categorization research. In this balanced spirit, the article's simulations are based on both kinds of category representation. Indeed, the analysis that shows most clearly the problem discussed in this article will feature the prototype $\mathrm{S}-\mathrm{C}$ model that I have used.

\section{Similarity and Choice in Categorization Models}

$\mathrm{S}-\mathrm{C}$ models take as their input the distance between a to-be-classified item (TBCI) and a category representation (CR). Then, the model (and presumably the mind) 
transforms distance into an estimated TBCI-CR similarity, and similarity into an estimated category endorsement level (i.e., an estimate of how strongly that item would be endorsed into its category). These transformations are described below.

Similarity. The distance input to $\mathrm{S}-\mathrm{C}$ models can be a featural difference (e.g., the stimuli 0000 and 0011 would be 2 apart). Alternatively, distance can incorporate attention (e.g., 0000 and 0011 would be .7 apart if .2 and .5 of attention went to Features 3 and 4, respectively) or reflect a well-studied psychological space (e.g., the space defined by Munsell coordinates). S-C models mathematically invert distance-less distance becomes more similarity-by making psychological similarity $\left(\operatorname{sim}_{\text {cat }}\right)$ an exponentialdecay function of distance (dist). A sensitivity parameter (sens) governs this inversion: $\operatorname{sim}_{\mathrm{cat}}=e^{- \text {sens } \times \text { dist }}$. Figure 1A shows this well-known relationship. TBCI-CR similarity increases more quickly as TBCI-CR distance decreases along the $x$-axis. This curve (increasing, curvilinear, and accelerating) is meant to incorporate the work of Shepard (e.g., 1987), who showed that participants' observed stimulus confusions were an exponential function of interstimulus distance.

Choice. S-C models then transform TBCI-CR similarity into a category endorsement level $\left(e l_{\text {cat }}\right)$. A criterion parameter, $\operatorname{sim}_{\text {null }}$, governs this transformation. The choice rule has the general form of $e l_{\text {cat }}=\operatorname{sim}_{\text {cat }} /\left(\operatorname{sim}_{\text {cat }}+\right.$ $\left.\operatorname{sim}_{\text {null }}\right)$. Figure 1B shows this relationship. Endorsement increases more slowly as similarity increases. This curve (increasing, curvilinear, and decelerating) is meant to incorporate the work of Luce (e.g., 1963).

These two graphs are purely mathematical and place no theory of category representation (e.g., prototype theory, exemplar theory) at risk. That is, these graphs would have the same character whether distance were reckoned to category exemplars or to category prototypes. In this sense, they give us theoretical safe harbor for examining the stages of the $\mathrm{S}-\mathrm{C}$ model in relation to one another. The problem is that the transformations of similarity and choice seem to be nearly inverses. At large distance/low similarity, similarity increases slowly but endorsement level increases quickly. At small distance/high similarity, similarity increases quickly but endorsement level slowly. The two functions compensate, suggesting that distance (the model's input) and endorsement level (the model's output) might relate linearly. Figure $1 \mathrm{C}$ confirms that they do: The $e l_{\text {cat }}-$ dist correlation is beyond .99 . Remarkably, the mathematical transformations cancel so that the model's input turns out to have been only linearly rescaled.

This cancellation phenomenon raises important questions regarding our interpretation of $\mathrm{S}-\mathrm{C}$ models and their ability to illuminate categorization. Would it be parsimonious to acknowledge the linear rescaling in addition to highlighting two nonlinear transformations? Given the linearity, would it sometimes be appropriate to use slope and intercept, rather than similarity and choice parameters, to describe the model's operation? Or would doing so compromise the $\mathrm{S}-\mathrm{C}$ model's relationship to other theories and models? How can we see and study similarity and choice within a model when these processes cancel? Where does the burden of proof lie in the claim that similarity and choice occur within a model when they cancel? Is the model overly complex for what it is doing? Does its complexity distract us from the psychologically valid information that may be contained within the distance inputs to the model?

Before discussing these issues, I explore whether or not this cancellation phenomenon determines the behavior and interpretation of S-C models in actual domains of category research.

\section{Simulation 1: A Single-Exemplar Category Representation}

This simulation adopted the influential dot distortion methodology (Knowlton \& Squire, 1993; Nosofsky \& Zaki, 1998; Palmeri \& Flanery, 1999; Posner, Goldsmith, \& Welton, 1967; Reber, Stark, \& Squire, 1998; Smith, 2002; Smith \& Minda, 2001, 2002). I assumed a TBCI at a
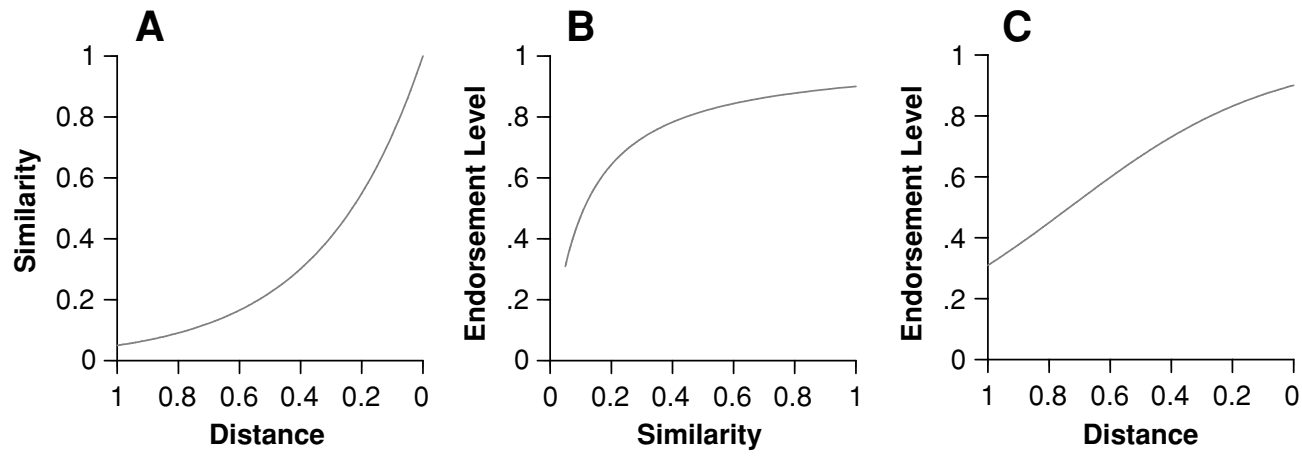

Figure 1. (A) An illustration of the similarity-distance relation in $\mathrm{S}-\mathrm{C}$ models. The similarity equation$\operatorname{sim}_{\mathrm{cat}}=e^{- \text {sens } \times \text { dist } — \text { was solved with sensitivity (sens) }}=3$ for distance 1.0 down to 0 . (B) An illustration of the endorsement level-similarity relation in $\mathrm{S}-\mathrm{C}$ models. The endorsement-level equation-el $-e$ $\operatorname{sim}_{\text {cat }} /\left(\operatorname{sim}_{\text {cat }}+\operatorname{sim}_{\text {null }}\right)$-was solved with criterion $\left(\operatorname{sim}_{\text {null }}\right)=.11111$ for similarity 0 up to 1.0. (C) An illustration of the endorsement level-distance relation in $\mathrm{S}-\mathrm{C}$ models, created by plotting endorsement level and distance directly. Figures $2-5$ have a similar structure. 
range of distances from a $\mathrm{CR}$ - from 0 (TCBI identical to the $\mathrm{CR}$ ) out to 2.89 . (This simulation has a prototype character because a prototype TBCI will have 0 distance from a prototype CR.) I transformed each distance into a similarity by choosing a random sensitivity $(0<\operatorname{sens}<1.77)$ and letting $\operatorname{sim}_{\text {cat }}=e^{- \text {sens }} \times$ dist . I transformed $\operatorname{sim}_{\text {cat }}$ into $e l_{\text {cat }}$ by choosing a random criterion $\left(0<\operatorname{sim}_{\text {null }}<0.44\right)$ and letting $e l_{\text {cat }}=\operatorname{sim}_{\text {cat }} /\left(\operatorname{sim}_{\text {cat }}+\operatorname{sim}_{\text {null }}\right)$. The ranges of these variables (distance, sensitivity, and criterion) covered the relevant ranges for modeling humans' dot distortion data. At each of 2,900,000 iterations (10,000 at every 0.01 distance from 0 to 2.89 ), the obtained similarity and endorsement level were stored in an array subscripted by the distance that produced them. Thus, I calculated the average similarity and endorsement level for each distance. (Individual configurations of the $\mathrm{S}-\mathrm{C}$ model are considered in Simulations 4-7.)

Figure 2 shows that the similarity (A) and endorsement (B) stages canceled to produce a linear rescaling (C). The $e l_{\text {cat }}-$ dist correlation was beyond .99 .

\section{Simulation 2: A 40-Exemplar Category Representation}

In each of the 32,000,000 iterations of Simulation 2, I chose 40 distortions of a prototype to be the category's exemplar-based CR. This simulation's exemplar character balances the previous simulation's prototype character. Across the iterations, I gradually increased the maximum distortion level possible for these exemplars (from 0 to the level allowed in dot-distortion studies) so that they gradually became more variable and spread out in psychological space. I chose one TBCI that was either a distortion of the category prototype at a random level or a high-level distortion of an unrelated prototype. I found the distance of the TBCI to every exemplar and averaged the 40 distances. I chose a random sensitivity from the same range as before, found the similarity of the TBCI to each exemplar, and averaged the 40 similarities. I chose a random criterion value from the same range as before and used the choice rule to find the endorsement level predicted from the average similarity. I stored similarities and endorsement levels in an array subscripted by the TBCI-CR distance that produced them. This analysis took $24 \mathrm{~h}$ on a fast PC.
Figure 3 duplicates Figure 2, with the stages of similarity (A) and endorsement level (B) canceling to produce a linear rescaling of distance $(\mathrm{C})$. The $e l_{\text {cat }}$-dist correlation was beyond .99 . Thus, regarding one of the most important categorization paradigms, the S-C model, with its two nonlinear stages, could be viewed as overly complex because a linear regression would serve as well mathematically.

\section{Simulation 3: Binary-Trait Categories}

Next, I turned to another principal domain of categorization research: binary-trait stimuli. I considered the case of four-dimensional stimuli arranged into two family resemblance categories containing the prototypes $(0000$, $1111)$ and the category members containing three typical traits (e.g., 0001, 0010 in Category A; 1110, 1101 in Category B). At each iteration, I chose a random attentional allocation for the $\mathrm{S}-\mathrm{C}$ model (i.e., attentional weights for the four dimensions that summed to 1.0) and a random sensitivity between 0.001 and 8.0 (knowing 8.0 to give a generous ceiling to this analysis). Given a configuration of the model, I found for the 10 stimuli the distance and similarity to each Category A and each Category B member, then the total distance and similarity to Category A and Category B, and, finally, the Category A endorsement level by applying the choice rule $e l_{\text {cat }}=\operatorname{sim}_{\text {catA }} /\left(\operatorname{sim}_{\text {catA }}+\right.$ $\left.\operatorname{sim}_{\text {catB }}\right)$. In the two-category case, $\operatorname{sim}_{\text {catB }}$ replaces $\operatorname{sim}_{\text {null }}$. The similarities and endorsement levels were stored in an array subscripted by the Total Category A distance that produced them. I evaluated 1,000,000 configurations of the model so as to end with $10,000,000$ stored observations.

Figure 4 shows again that choice (B) undid similarity (A). The $e l_{\text {cat }}-$ dist correlation was .99 (C). Thus, regarding another important categorization paradigm, the $\mathrm{S}-\mathrm{C}$ model, with its two nonlinear stages, could be viewed as overly complex because a regression would serve as well mathematically. This is potentially a serious problem because dot-distortion and binary-trait studies combined represent an important segment of the categorization literature.

\section{Simulations 4-7: Individual Performance Configurations}

A concern about Simulations $1-3$ is that I inferred the S-C model's operating characteristics from its average
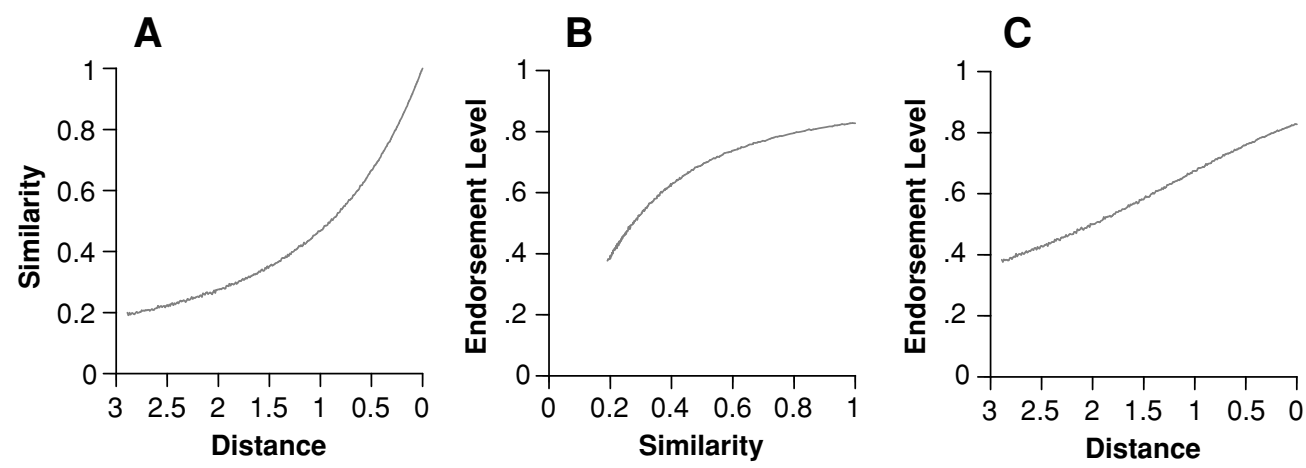

Figure 2. Results of Simulation 1. 

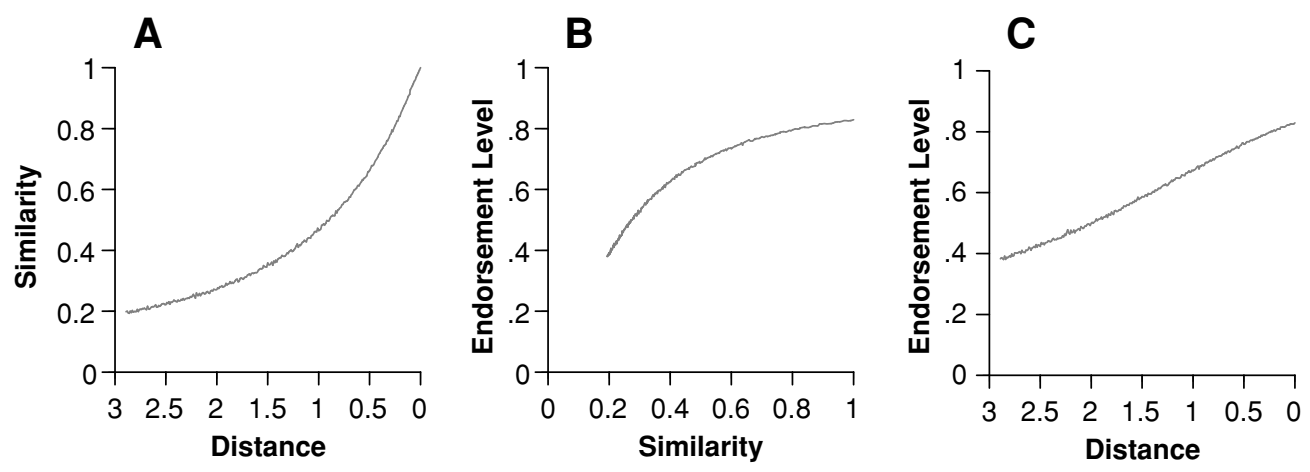

Figure 3. Results of Simulation 2.

behavior when the purpose of modeling is to find particular model configurations that fit particular data. (It is an interesting question whether one can glean insights about models from their average behavior.) I addressed this concern by examining individual configurations of the $\mathrm{S}-\mathrm{C}$ model.

In Simulation 4, I focused on the item types - prototype, low-level distortion, high-level distortion, and randomthat are tested in dot-distortion studies. I used established values for the distances between training exemplars and these item types. These distances were 1.76, 1.87, 2.1, and 2.89, respectively (Smith, 2002). I chose sensitivity and criterion values from the same ranges as before and found, for each TBCI type, its similarity to the training items (by transforming the distance) and its category endorsement level (by transforming the similarity). Finally, I found the linear correlation between endorsement level and distance for each model configuration. Across 10,000 configurations, the average $e l_{\text {cat }}-$ dist correlation was .99 $(S D=.005)$. The cancellation of similarity by choice extends to individual model configurations.

In Simulation 5, I examined 10,000 individual configurations of the $\mathrm{S}-\mathrm{C}$ model in the binary-trait domain. For each, I chose random attentional weights and sensitivity. Given a configuration of the model, I found the category distance, similarity, and endorsement level for each of the 10 stimuli in the way already described. Finally, I found the linear correlation between endorsement level and distance for each configuration of the model. Across the 10,000 configurations, the average correlation was .99 $(S D=.015)$.

Simulations 6 and 7 were re-creations of Simulations 4 and 5, respectively, covering the $\mathrm{S}-\mathrm{C}$ model's entire parameter space. For the dot-distortion case, I examined 1,540 model configurations that varied sens from 0.05 to 1.75 in .05 increments and $\operatorname{sim}_{\text {null }}$ from 0.01 to 0.44 in .01 increments. For the binary-trait case, I examined 2,228 model configurations that varied sens from 1 to 8 in 1.0 increments and the four attentional parameters from 0 to 1.0 in .1 increments. Both of the average $e l_{\text {cat }}$-dist correlations were still $.99(S D \mathrm{~s}=.005$ and .018 for Simulations 6 and 7, respectively), showing that the S-C cancellation extends across the S-C model's whole parameter space.

\section{Simulation 8: Simple Mathematics}

Simulation 8 explains why the cancellation phenomenon is a mathematical fact apart from any competition between theories. A categorization task always has worst and best items with, respectively, maximum and minimum CR distances, minimum and maximum CR similarities (mediated by sens), and minimum and maximum endorsement levels (mediated by $\operatorname{sim}_{\text {null }}$ ). Table 1 illustrates this framework, assuming worst and best items with 1 and $0 \mathrm{CR}$ distance, endorsement levels of .1 and .9 for
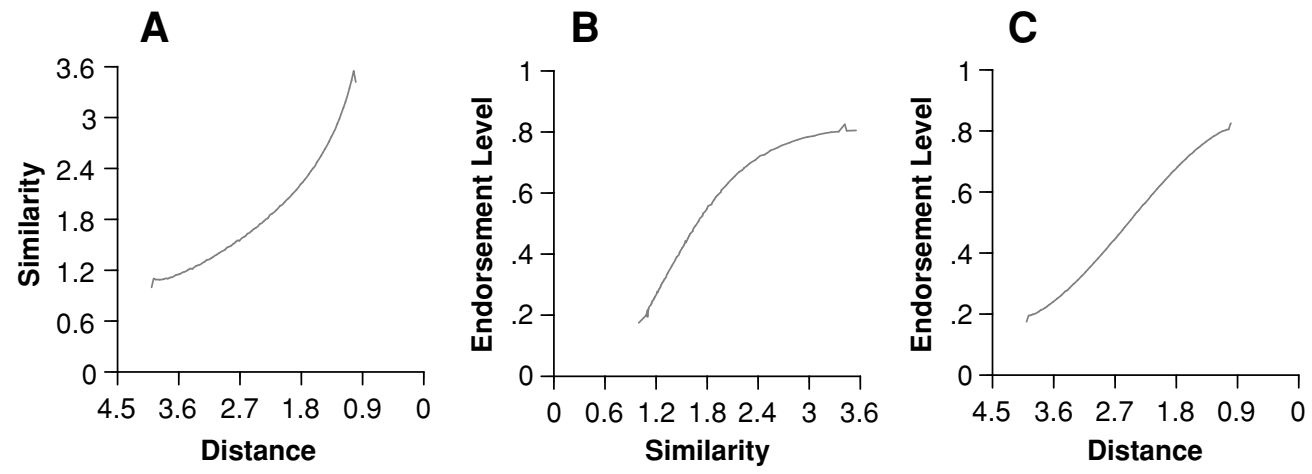

Figure 4. Results of Simulation 3. 
Table 1

Structure, Calculations, and Constraints of the S-C Model

\begin{tabular}{cccccc}
\hline $\begin{array}{c}\text { Stimulus } \\
\text { Type }\end{array}$ & CR Distance & $\begin{array}{c}\text { Sensitivity } \\
\text { Parameter } \\
(\text { sens })\end{array}$ & CR Similarity & $\begin{array}{c}\text { Choice Rule } \\
\text { Criterion } \\
\left(\text { sim }_{\text {null }}\right)\end{array}$ & $\begin{array}{c}\text { Endorsement } \\
\text { Level }\end{array}$ \\
\hline Worst & 1.0 & & $\mathbf{0 . 0 1 2 3 4 5 6 7 9}$ & $\mathbf{0 . 1 1 1 1 1 1 1 1 1}$ & .1 \\
Best & 0.0 & 4.394449156 & 1.000000000 & & .9 \\
\hline
\end{tabular}

Note-CR, category representation.

these items (because to err is human), and assuming that 0 distance maps to 1 (perfect) similarity. One can use the choice rule- $e l_{\text {cat }}=\operatorname{sim}_{\text {cat }} /\left(\operatorname{sim}_{\text {cat }}+\operatorname{sim}_{\text {null }}\right)$ - to determine that $\operatorname{sim}_{\text {null }}$ in this case is $0.111111111[.9=1 /(1+$ $0.111111111)]$. One can determine that the worst item's CR similarity is $0.012345679[.1=0.012345679 /(0.0123$ $45679+0.111111111)]$. Furthermore, one can determine that sensitivity for this example must be 4.394449156 $\left(e^{-1 \times 4.394449156}=0.012345679\right)$. Given sens and $\operatorname{sim}_{\text {null }}$, one can vary distance over its range, find corresponding similarities and endorsement levels, and thus find that the $e l_{\text {cat }}$-dist correlation is .99 .

This procedure can be automated to produce many cases. I let worst distance vary from 1.0 to 4.0 , worst endorsement level vary from .01 to .20 , and best endorsement level vary from .80 to .99 . I kept best distance at 0 and best similarity at 1 for intuitive reasons. Sens and $\operatorname{sim}_{\text {null }}$ followed analytically, as did the $e l_{\text {cat }}-$ dist correlation over each case's range of variables. The average $e l_{\text {cat }}$-dist correlation was .99 over 10,000 randomly chosen cases. The simulations in this article have no theoretical slant. In important domains of categorization that constitute most of the existing literature, the cancellation of similarity by choice is simply mathematical over the performance levels humans show and the attendant ranges of similarity and distance.

\section{Simulation 9: When Distance and Similarity Dissociate}

S-C modelers might point to my focus on family resemblance categories in which distance and similarity do not dissociate strongly. Perhaps these examples do not show the $\mathrm{S}-\mathrm{C}$ model in its best light. Thus, it is important to con- sider a case of dissociation that delimits the phemonemon treated in this article. To do so, I used the well-known 5-4 category structure (see, e.g., Blair \& Homa, 2003; Smith \& Minda, 2000). In this category structure, the A stimuli are 1110,1010, 1011, 1101, and 0111; the B stimuli are $1100,0110,0001$, and 0000; and the transfer stimuli are 1001, 1000, 1111, 0010, 0101, 0011, and 0100.

In each iteration, I chose a random attentional allocation for the $\mathrm{S}-\mathrm{C}$ model and a random sensitivity (0.001-8.0). I found the distance, similarity, and endorsement level for the 16 stimuli as described in Simulation 3. The 16 similarity and endorsement levels were stored in an array subscripted by the Total Category A distance so that I could calculate the similarity and endorsement level for each distance. I evaluated 1,000,000 configurations of the S-C model to end with $16,000,000$ stored observations.

Figure 5 shows the result. Similarity and distance do dissociate in this task. Panel A shows that some largedistance stimuli have surprisingly high CR similarity. This effect is caused mainly by configurations of the model in which the model assumes nearly singular attention to only the first dimension. The $e l_{\text {cat }}-\operatorname{sim}_{\text {cat }}$ plot (panel B) maintains this dissociation - there is a kink in the function. But the two curves are still complementary. The $e l_{\text {cat }}$-dist correlation was .98 (panel C), demonstrating that choice still powerfully unraveled similarity.

This is an intriguing case. It is as though we had bent a rope into an exponential-decay shape, but it had a kink. The choice rule yanked the rope straight but left the kink. Thus, the main effect of exponentiation was removed, but perhaps something interesting remained. The problem is that we don't know what left and what stayed. What stayed is not, and should not be called, Shepard exponentiation.
A

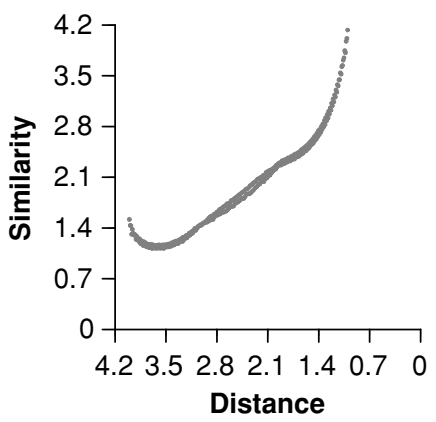

B

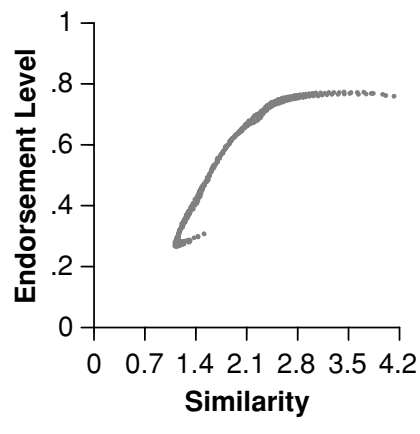

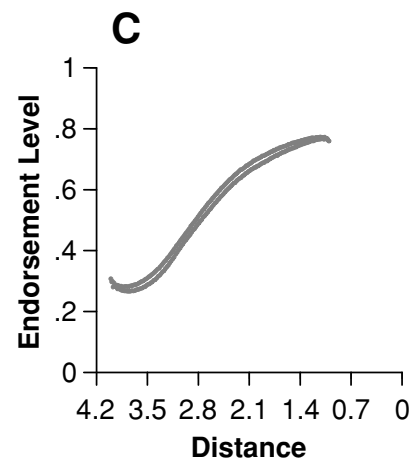

Figure 5. Results of Simulation 9. 
But what is it? Even if one thinks that similarity and choice are important components of categorization, the cancellation phenomenon makes it difficult to see them or the shape of their underlying psychological functions.

\section{Simulation 10: Alternative Parameterizations of Performance Space}

Earlier, I asked whether or not slope-intercept descriptions of the $\mathrm{S}-\mathrm{C}$ model's operation might be equivalent in some ways to $\mathrm{S}-\mathrm{C}$ descriptions. A final simulation helped me make this comparison directly. I examined 14,641 (114) hypothetical dot-distortion performance profiles, across which endorsement varied, in eleven .05 steps, from .50 to 1.00 (prototypes), from .40 to .90 (low-level distortions), from .30 to .80 (high-level distortions), and from .20 to .70 (random, unrelated items). I let the exemplar S-C model fit each profile, using the distance inputs described in Simulation 4. This yielded a sensitivity value and a criterion value for each performance profile. I also regressed the model's predictions onto the distance inputs. This yielded a slope and an intercept value for each performance profile. The 14,641 data tetrads offered-in parameter pairs - two descriptions of how distance relates to performance through the model.

Table 2 shows the correlations among the slope, intercept, sensitivity, and criterion values across the data tetrads. Clearly, slope and sensitivity were very highly related: Their actual correlation was -.997 . However, all the intercorrelations were strong. I analyzed the tetrads with the factor analytic techniques in SAS 9.0. Using the principal factors method, the first factor explained $98 \%$ of the variance in the four measures. Its eigenvalue was 3.66 , in comparison with 0.08 for the second factor. This analysis shows that really only one thing varies across performance profiles, with all four parameters entrained to it, even if the slope-intercept and sensitivity-criterion descriptions offer alternative viewpoints on what that thing is. This simulation reinforces Simulation 8 in showing that the mapping from distance to performance in the $\mathrm{S}-\mathrm{C}$ model is simple and almost analytic, so that this mapping may not bear the psychological weight and complexity we normally confer upon it.

\section{Discussion}

This article contains a negative message about $\mathrm{S}-\mathrm{C}$ models that applies whether they are based in prototype or in exemplar theories of category representation. One response would be to narrow the application of these models to domains in which the cancellation problem is less

Table 2

Comparison of Slope-Intercept and Sensitivity-Criterion Parameterizations of the Performance Space of the S-C Model

\begin{tabular}{lcccc}
\hline & Slope & Intercept & Sensitivity & Criterion \\
\hline Slope & - & -.97 & -.99 & .82 \\
Intercept & & - & .97 & -.87 \\
Sensitivity & & & - & -.82 \\
Criterion & & & & - \\
\hline
\end{tabular}

severe. That is, one might agree that these models are less appropriate for dot-distortion and binary-trait data because they are unnecessarily complex for those contexts. However, this resolution of the problem fails for several reasons. The majority of the $\mathrm{S}-\mathrm{C}$ model's applications have concerned those domains - casting doubt on them would damage the model's importance and utility and leave it applicable only to the literature's stranger categories. Moreover, in the end, this exclusion by domain would not even work. The cancellation of similarity by choice is mathematically inexorable and will always apply (Simulation 8), even when distance and similarity dissociate (Simulation 9). Essentially all category structures would show a powerful cancellation effect, and a 90\% cancellation would almost be worse than the $99 \%$ cancellation illustrated here, for then one would know less clearly what the model had canceled out or left behind, whereas with perfect cancellation at least one sees the linear rescaling.

Nor is it a strongly saving point that these linearities sometimes flatten slightly in their extreme ranges, as though perfect cancellation might not apply given extreme enough category members. Although this is true, and although one might choose to study the psychology of extreme category members, the category structures used in the present simulations encompass fairly and exactly the categories upon which the field was founded and around which it was built. The cancellation phenomenon is nearly perfect regarding the existing data on these categories and $\mathrm{S}-\mathrm{C}$ models of those data.

Even while endorsing this article's demonstrations, reviewers raised fruitful questions about formal models and the preferred parameterization of their prediction spaces. They argued that the parameters of slope and intercept are psychologically poorer than the constructs of similarity and choice. They argued that one might prefer to retain the $\mathrm{S}-\mathrm{C}$ parameterization, given the conceptual connections of these parameters to other research traditions and given the hope that these parameters would let one see independent effects on similarity and choice caused by development, aging, expertise, category structure, and so forth-effects that one might not show as interestingly using slope and intercept.

These issues deserve consideration by the community of categorization researchers in particular and by modelers more broadly. I am not prescribing the use of slopes and intercepts. I am simply describing the structural dynamics of an important categorization model. Setting the $\mathrm{S}-\mathrm{C}$ model aside for a moment, I would generally agree that slopes and intercepts are less rich psychologically than grounded constructs of similarity and choice (if these could be instantiated in a model), and I would generally endorse the hope of making theoretical connections to other domains.

However, the standards of science and parsimony suggest that S-C models do not fulfill this hope. If objects fell at a constant velocity, it would probably not be acceptable to explain this by assuming two nonlinear, canceling forces in nature (gravity and force Z). If pigeons' rate of pecking increased linearly with wavelength, it would probably not 
be acceptable to explain this by using two unobservable, nonlinear transformations. To the extent that $\mathrm{S}-\mathrm{C}$ models also posit unobservable, canceling transformations, there is reason to bring a similar caution to their description of categorization.

In fact, neither similarity nor choice is measured separately or observably in the $\mathrm{S}-\mathrm{C}$ model. They are only estimated within the overall model-fitting procedure that includes both components. Exponential-decay similarity - the hypothetical midpoint of the S-C model's calculations - was introduced into the model without reference to Shepard's (1987) work (Medin \& Schaffer, 1978; Medin \& Smith, 1981); these connections were drawn later. Moreover, whereas Shepard's exponentialdecay similarity was grounded on observable confusions between stimuli, similarity in the $\mathrm{S}-\mathrm{C}$ model is not. Likewise, the choice stage was included in the $\mathrm{S}-\mathrm{C}$ model not as a psychologically grounded process but as a convenience (Medin \& Smith, 1981, p. 250) for researchers, who responsibly acknowledged that it was an approximation used because "it seems to work." Note also that the choice rule plays a dual and ambiguous role in the $\mathrm{S}-\mathrm{C}$ model. It may be present in the model as much for its mathematical function (rescaling the inputs into $0 \%-100 \%$ performance measures) as for its psychological function. Finally, note that in many applications of the model (i.e., those involving more than one category), the choice stage does not even have an independent criterion parameter. Instead, the choice rule uses the opposing-category similarity (see Simulation 3 above), ensuring dependence between the operation of choice and the estimation of similarity.

Given the simultaneous and interdependent estimation of similarity and choice during fitting, the $\mathrm{S}-\mathrm{C}$ cancellation is a significant obstacle to forging separate and theoretical links to the literatures on perceptual similarity and decisional choice. How can we see the separate contribution of Shepard exponentiation to categorization or its independent relation to other areas when its effects are so well nullified by choice? If the sensitivity parameter in the similarity calculation increases to a high level, is this because we have a sensitive perceiver or because the high sensitivity level was compensating for the canceling effect of choice? This is a difficult problem no matter how friendly one is to the $\mathrm{S}-\mathrm{C}$ model's individual components. It is useful history to know that the choice rule and the exponential similarity calculation were combined in S-C models of categorization from the first uses of these models. This may explain why the potential problems with these models were difficult for well-meaning, groundbreaking researchers to discern. But it also suggests that the problems were present from the beginning.

We all share the goal of illuminating the psychological representations and processes that underlie humans' categorization behavior. Without prescribing anything, it does seem that two unobservable, nonlinear transformations that cancel to produce a linear rescaling may not be very helpful in furthering this goal. We should at least blend this complementary perspective on the $\mathrm{S}-\mathrm{C}$ model into our thinking about it.
This discussion, and the present article's demonstrations, help explain why $\mathrm{S}-\mathrm{C}$ models have had difficulty showing independent effects relating similarity, choice, and their parameter values to other variables and difficulty making a priori predictions generally (Maddox \& Ashby, 1998), whereas other modeling perspectives have been able to do so (e.g., Ell \& Ashby, 2004). This discussion also helps explain why there is a current concern that the S-C-model framework has tipped too steeply toward the mathematical fitting of data and too steeply against instantiating psychological processes conceptually and transparently (Maddox \& Ashby, 1998; Minda \& Smith, 2001). To these concerns, the present article adds that the S-C model's components are caught in a mathematical interaction that does not allow them to stand free as psychological processes and stages even if the idea of those processes and stages is intuitively appealing.

But this does not mean that similarity and choice inevitably cancel or are inherently unworkable within a modeling framework. There might be ways to measure similarity and choice independently, much as signal detection researchers separate sensitivity and bias. One might then study their independent effects and correlates, arrange dissociations between them, and so forth. As an illustrative approach to measurement, one could collect similarity ratings on pairs of stimuli and consider these a grounded measure of psychological similarity. ${ }^{1}$ Further advancements in studying psychological similarity and choice may assist in the development of such a framework (see, e.g., Chater \& Vitányi, 2003). It is reasonable to view categorization as involving similarity and choice, and it is a constructive wish to study similarity and choice as independent aspects of categorization. But the $\mathrm{S}-\mathrm{C}$ model is not a framework that allows that study.

Nor does this discussion imply that all conflicting mathematical transformations must be rooted out of formal models. Hurricanes are fueled by warm Gulf water but weakened by high-altitude shearing winds. These observable, independent forces are canceling, but nonetheless real and grounded independently and in their interaction. Cognition is probably as messy as weather, so process cancellations may happen there, too. The problem dealt with in the present article arises when a model does a simple thing, but we call it a complex and psychologically staged thing even though those stages are not grounded or observable and even though they are not necessary to explain what the model does.

Pursuing this idea further, another approach to modeling categorization would be to incorporate the transformations that occur in the brain during categorization, including, for example, the mapping of visual cortical cells to striatum. Then, if we discovered canceling transformations, we would at least know that they were "our" canceling transformations. Even if this approach did not produce independent stages of similarity and choice, it would be grounded and revealing about how mind and brain accomplish the categorization goal. Greg Ashby and his colleagues are breaking important ground in this area (see, e.g., Ashby, Ell, Valentin, \& Casale, 2005). 
Table 3

Illustration of a Positive Message About Psychological Distances

\begin{tabular}{lcccc}
\hline & \multirow{2}{*}{$\begin{array}{c}\text { Amnesics' } \\
\text { Stimulus Type }\end{array}$} & \multicolumn{3}{c}{ Prototype } \\
\cline { 3 - 5 } \cline { 3 - 5 } Performance* & Distance & Model & Regression \\
Low-level distortion & .73 & 0.00 & .72 & .72 \\
High-level distortion & .60 & 1.09 & .62 & .62 \\
Random, unrelated & .57 & 1.76 & .56 & .56 \\
& .46 & 2.85 & .46 & .46 \\
\hline
\end{tabular}

*Data from Knowlton and Squire (1993).

This article also contains an important positive message about illuminating categorization: The distances we input into our categorization models are often sufficiently grounded psychologically to predict categorization directly with no nonlinear transformations applied at all. I will illustrate this positive message now.

Knowlton and Squire (1993) found that when amnesics endorsed dot patterns into categories, they showed a strong prototype enhancement effect (leftmost data column of Table 3). This could mean that amnesics have intact prototype abstraction processes despite damaged exemplar memory systems (see the discussions in Knowlton \& Squire, 1993; Nosofsky \& Zaki, 1998; Smith, 2002; Smith \& Minda, 2001). One approach to this issue has been to apply the S-C model, inputting into it the prototype-TBCI distances (second column of Table 3), then using $\operatorname{sim}_{\text {cat }}$ and $\operatorname{sim}_{\text {null }}$ to find the $\mathrm{S}-\mathrm{C}$ model's best-fitting predictions (third column of Table 3). The distances in Table 3 were calculated using established, psychophysically validated procedures - found in Posner et al. (1967) and Smith and Minda (2001, 2002) - that involve the Pythagorean distance over which dots are moved between patterns. The model fits the data beautifully. However, the table's fourth column shows the result of merely regressing amnesics' category endorsements onto the distances. Comparing the third and fourth columns, one sees that, for all its complexity and gravitas, the $\mathrm{S}-\mathrm{C}$ model once more offered only a linear regression because of the $\mathrm{S}-\mathrm{C}$ collision. Anyone interested in the S-C-model framework should attend closely to this demonstration. It is incidental that these distances were prototype based. Indeed, this example is deliberately critical of my use of prototype S-C models (Smith \& Minda, 2001). The point is that the distances came through the model linearly rescaled but otherwise unscathed.

Positively, though, this demonstration shows that the unadorned distance relations among stimuli carry valuable information regarding categorization if these relations are well conceived. It shows that the distances contain the psychological information necessary for predicting categorization. This fact may honor Shepard's (1987) ideas more than does using his exponential curve in a canceling model. His overarching point was that if one got the psychological distances among stimuli right, one could explain many things. Here, on getting the distances right, we saw, with no modeling, no fitting, and linearly, that the distance measures embodied the psychologically true category representation and the empirically true endorsement levels. This analysis shows that, as we strip from our models unnecessary transformations, the models may come to reflect better the psychology of participants. And this is the goal of our models.

My final point is for modelers generally. Models have grown complex as programming and computation have developed. Parameters and mathematical transformations are added to models freely and pragmatically (as when activation functions constrain neural net models within good working limits). Many models risk the kind of interaction demonstrated here. The risk increases as the model becomes more complex and includes more nonpsychological transformations. The risk is unknown if the relationships among transformations are not queried. Indeed, the present internal analysis uncovered an interaction that had gone unnoticed in an influential model. One source of protection lies in carrying out similar internal analyses for other models of interest. Another lies in keeping models as simple, psychological, and transparent as possible. In fact, this is the best way to avoid the collision of parameters that $\mathrm{S}-\mathrm{C}$ models illustrated here.

\section{REFERENCES}

Ashby, F. G., Ell, S. W., Valentin, V. V., \& Casale, M. B. (2005). FROST: A distributed neurocomputational model of working memory maintenance. Journal of Cognitive Neuroscience, 17, 1728-1743.

Blair, M., \& Homa, D. (2003). As easy to memorize as they are to classify: The 5-4 categories and the category advantage. Memory \& Cognition, 31, 1293-1301.

Chater, N., \& Vitányi, P. M. B. (2003). The generalized universal law of generalization. Journal of Mathematical Psychology, 47, 346-369.

Ell, S. W., \& AshBY, F. G. (2004). Dynamical trajectories in category learning. Perception \& Psychophysics, 66, 1318-1340.

Knowlton, B. J., \& Squire, L. R. (1993). The learning of categories: Parallel brain systems for item memory and category knowledge. Science, 262, 1747-1749.

LucE, R. D. (1963). Detection and recognition. In R. D. Luce, R. R. Bush, \& E. Galanter (Eds.), Handbook of mathematical psychology (Vol. 1, pp. 103-189). New York: Wiley.

Maddox, W. T., \& Ashby, F. G. (1998). Selective attention and the formation of linear decision boundaries: Comment on McKinley and Nosofsky (1996). Journal of Experimental Psychology: Human Perception \& Performance, 24, 301-321.

Medin, D. L., \& Schaffer, M. M. (1978). Context theory of classification learning. Psychological Review, 85, 207-238.

Medin, D. L., \& Smith, E. E. (1981). Strategies and classification learning. Journal of Experimental Psychology: Human Learning \& Memory, 7, 241-253.

Minda, J. P., \& Smith, J. D. (2001). Comparing prototype-based and exemplar-based accounts of category learning and attentional allocation. Journal of Experimental Psychology: Learning, Memory, \& Cognition, 28, 275-292.

Nosofsky, R. M., \& ZAKI, S. R. (1998). Dissociations between categorization and recognition in amnesic and normal individuals: An exemplar-based interpretation. Psychological Science, 9, 247-255.

Palmeri, T. J., \& Flanery, M. A. (1999). Learning about categories 
in the absence of training: Profound amnesia and the relationship between perceptual categorization and recognition memory. Psychological Science, 10, 526-530.

Posner, M. I., Goldsmith, R., \& Welton, K. E., JR. (1967). Perceived distance and the classification of distorted patterns. Journal of Experimental Psychology, 73, 28-38.

Reber, P. J., Stark, C. E. L., \& SQuire, L. R. (1998). Cortical areas supporting category learning identified using functional MRI. Proceedings of the National Academy of Science, 95, 747-750.

Roberts, S., \& Pashler, H. (2000). How persuasive is a good fit? A comment on theory testing. Psychological Review, 107, 358-367.

SHEPARD, R. N. (1987). Toward a universal law of generalization for psychological science. Science, 237, 1317-1323.

SMITH, J. D. (2002). Exemplar theory's predicted typicality gradient can be tested and disconfirmed. Psychological Science, 13, 437-442.

SMith, J. D., \& MindA, J. P. (2000). Thirty categorization results in search of a model. Journal of Experimental Psychology: Learning, Memory, \& Cognition, 26, 3-27.

Smith, J. D., \& MindA, J. P. (2001). Journey to the center of the cat- egory: The dissociation in amnesia between categorization and recognition. Journal of Experimental Psychology: Learning, Memory, \& Cognition, 27, 984-1002.

SMITH, J. D., \& Minda, J. P. (2002). Distinguishing prototype-based and exemplar-based processes in dot-pattern category learning. Journal of Experimental Psychology: Learning, Memory, \& Cognition, 28, 800-811.

\section{NOTE}

1. Although $\mathrm{S}-\mathrm{C}$ modelers often collect similarity ratings in this way, these are then input into a multidimensional-scaling routine that transforms them into dimensionalized distances that are then used to estimate exponential-decay similarity among stimuli during combined S-C model fitting. In a sense, this procedure turns observable, independent similarity into unobservable, dependent similarity.

(Manuscript received February 3, 2005; revision accepted for publication February 11, 2006.) 